УДК 517.95

\title{
Rigidity Conditions for the Boundaries of Submanifolds in a Riemannian Manifold
}

\author{
Anatoly P. Kopylov* \\ Mikhail V. Korobkov ${ }^{\dagger}$ \\ Sobolev Institute of Mathematics SB RAS \\ 4 Acad. Koptyug avenue, Novosibirsk, 630090 \\ Novosibirsk State University \\ Pirogova, 2, Novosibirsk, 630090
}

Russia

Received 20.03.2016, received in revised form 28.04.2016, accepted 26.05.2016

Developing A.D. Aleksandrov's ideas, the first author proposed the following approach to study of rigidity problems for the boundary of a $C^{0}$-submanifold in a smooth Riemannian manifold. Let $Y_{1}$ be a two-dimensional compact connected $C^{0}$-submanifold with non-empty boundary in some smooth twodimensional Riemannian manifold $(X, g)$ without boundary. Let us consider the intrinsic metric (the infimum of the lengths of paths, connecting a pair of points".) of the interior Int $Y_{1}$ of $Y_{1}$, and extend it by continuity (operation $\underline{\mathrm{lm}}$ ) to the boundary points of $\partial Y_{1}$. In this paper the rigidity conditions are studied, i.e., when the constructed limiting metric defines $\partial Y_{1}$ up to isometry of ambient space $(X, g)$. We also consider the case $\operatorname{dim} Y_{j}=\operatorname{dim} X=n, n>2$.

Keywords: Riemannian manifold, intrinsic metric, induced boundary metric, strict convexity of submanifold, geodesics, rigidity conditions.

DOI: 10.17516/1997-1397-2016-9-3-320-331.

\section{Introduction: unique determination of surfaces by their relative metrics on boundaries}

A classical theorem says (see [3]): If two bounded closed convex surfaces in the threedimensional Euclidean space are isometric in their intrinsic metrics then they are equal, i.e., they can be matched by a motion.

The problems of unique determination of closed convex surfaces by their intrinsic metrics goes back to the result of Cauchy, obtained already in 1813, that any closed convex polyhedrons $P_{1}$ and $P_{2}$ (in the three-dimensional Euclidean space) that are equally composed of congruent faces are equal. Since then this problem has been studied by many people for about 140 years (for example, by Minkowski, Hilbert, Weyl, Blaschke, Cohn-Vossen, Aleksandrov, Pogorelov and other prominent mathematicians (see, for instance, the historical overview in [3], Chapter 3); finally, its complete solution, which is just the theorem we have cited at the beginning, was obtained by A. V. Pogorelov. For generalizations of Pogorelov's result to higher dimensions, see [4].

In [5], we proposed a new approach to the problem of unique determination of surfaces, which enabled us to substantially enlarge the framework of the problem. The following model situation illustrates the essence of this approach fairly well:

\footnotetext{
*apkopylov@yahoo.com

†korob@math.nsc.ru

(C) Siberian Federal University. All rights reserved
} 
Let $U_{1}$ and $U_{2}$ be two domains (i.e., open connected sets) in the real $n$-dimensional Euclidean space $\mathbb{R}^{n}$ whose closures $\mathrm{cl} U_{j}$, where $j=1,2$, are Lipschitz manifolds (such that $\partial\left(\operatorname{cl} U_{j}\right)=$ $\partial U_{j} \neq \varnothing$, where $\partial E$ is the boundary of $E$ in $\mathbb{R}^{n}$ ). Assume also that the boundaries $\partial U_{1}$ and $\partial U_{2}$ of these domains, which coincide with the boundaries of the manifolds $\mathrm{cl} U_{1}$ and $\operatorname{cl} U_{2}$, are isometric with respect to their relative metrics $\rho_{\partial U_{j}, U_{j}}(j=1,2)$, i.e., with respect to the metrics that are the restrictions to the boundaries $\partial U_{j}$ of the extensions $\rho_{\mathrm{cl} U_{j}}$ (by continuity) of the intrinsic metrics $\rho_{U_{j}}$ of the domains $U_{j}$ to $\mathrm{cl} U_{j}$. The following natural question arises: Under which additional conditions are the domains $U_{1}$ and $U_{2}$ themselves isometric (in the Euclidean metric)? In particular, the natural character of this problem is determined by the circumstance that the problem of unique determination of closed convex surfaces mentioned at the beginning of the article is its most important particular case. Indeed, assume that $S_{1}$ and $S_{2}$ are two closed convex surfaces in $\mathbb{R}^{3}$, i.e., they are the boundaries of two bounded convex domains $G_{1} \subset \mathbb{R}^{3}$ and $G_{2} \subset \mathbb{R}^{3}$. Let $U_{j}=\mathbb{R}^{3} \backslash \operatorname{cl} G_{j}$ be the complement of the closure $\operatorname{cl} G_{j}$ of the domain $G_{j}$, $j=1,2$. Then the intrinsic metrics on the surfaces $S_{1}=\partial U_{1}$ and $S_{2}=\partial U_{2}$ coincide with the relative metrics $\rho_{\partial U_{1}, U_{1}}$ and $\rho_{\partial U_{2}, U_{2}}$ on the boundaries of the domains $U_{1}$ and $U_{2}$, and thus the problem of unique determination of closed convex surfaces by their intrinsic metrics is indeed a particular case of the problem of unique determination of domains by the relative metrics on their boundaries.

The generalization of the problem of the unique determination of surfaces ensuing from a new approach suggested in [5] manifests itself in the fact that the unique determination of domains by the relative metrics on their boundaries holds not only when their complements are bounded convex sets but, for example, also in the following cases.

The domain $U_{1}$ is bounded and convex and the domain $U_{2}$ is arbitrary (A.P. Kopylov [5]).

The domain $U_{1}$ is strictly convex and the domain $U_{2}$ is arbitrary (A. D. Aleksandrov (see [6])).

The domains $U_{1}, U_{2}$ are bounded and their boundaries are smooth (V. A. Aleksandrov [6]).

The domains $U_{1}$ and $U_{2}$ have nonempty bounded complements, while their boundaries are $(n-1)$-dimensional connected $C^{1}$-manifolds without boundary, $n>2$ (V. A. Aleksandrov [7]).

In the papers [8-10], M.V. Korobkov (in particular) obtained a complete solution to the problem of unique determination of a plane (space) domain in the class of all plane (space) domains by the relative metric on its boundary.

In this connection, there appears the following question: Is it possible to construct an analog of the theory of rigidity of surfaces in Euclidean spaces in the general case of the boundaries of submanifolds in Riemannian manifolds?

Our article is devoted to a detailed discussion of this question. In it, we in particular obtain new results concerning rigidity problems for the boundaries of $n$-dimensional connected submanifolds with boundary in $n$-dimensional smooth connected Riemannian manifolds without boundary $(n \geqslant 2)$.

In what follows, all paths $\gamma:[\alpha, \beta] \rightarrow \mathbb{R}^{n}$, where $\alpha, \beta \in \mathbb{R}$, are assumed continuous and non-constant, and $l(\gamma)$ means the length of a path $\gamma$.

\section{Rigidity problems and intrinsic geometry of submanifolds in riemannian manifolds}

Let $(X, g)$ be an $n$-dimensional smooth connected Riemannian manifold without boundary and let $Y$ be an $n$-dimensional compact connected $C^{0}$-submanifold in $X$ with nonempty boundary $\partial Y(n \geqslant 2)$.

A classical object of investigations (see, for example, [11]) is given by the intrinsic metric $\rho_{\partial Y}$ on the hypersurface $\partial Y$ defined for $x, y \in \partial Y$ as the infimum of the lengths of curves $\nu \subset \partial Y$ joining $x$ and $y$. In the recent decades, an alternative approach arose in the rigidity theory for submanifolds of Riemannian manifolds (see, for instance, the recent articles [1,10], and [2], which 
also contain a historical survey of works on the topic). In accordance with this approach, the metric on $\partial Y$ is induced by the intrinsic metric of the interior Int $Y$ of the submanifold $Y$.

Namely, suppose that $Y$ satisfies the following condition:

(i) if $x, y \in Y$, then

$$
\rho_{Y}(x, y)=\liminf _{x^{\prime} \rightarrow x, y^{\prime} \rightarrow y ; x^{\prime}, y^{\prime} \in \operatorname{Int} Y}\left\{\inf \left[l\left(\gamma_{x^{\prime}, y^{\prime}, \operatorname{Int} Y}\right)\right]\right\}<\infty,
$$

where $\inf \left[l\left(\gamma_{x^{\prime}, y^{\prime}, \text { Int } Y}\right)\right]$ is the infimum of the lengths $l\left(\gamma_{x^{\prime}, y^{\prime}, \text { Int } Y}\right)$ of smooth paths $\gamma_{x^{\prime}, y^{\prime}, \text { Int } Y}$ : $[0,1] \rightarrow \operatorname{Int} Y$ joining $x^{\prime}$ and $y^{\prime}$ in the interior $\operatorname{Int} Y$ of $Y$.

Remark 2.1. Easy examples show that if $X$ is an $n$-dimensional connected smooth Riemannian manifold without boundary then an $n$-dimensional compact connected $C^{0}$-submanifold in $X$ with nonempty boundary may fail to satisfy condition (i). For $n=2$, we have the following counterexample:

Let $(X, g)$ be the space $\mathbb{R}^{2}$ equipped with the Euclidean metric and let $Y$ be a closed Jordan domain in $\mathbb{R}^{2}$ whose boundary is the union of the singleton $\{0\}$ consisting of the origin 0 , the segment $\left\{(1-t)\left(e_{1}+2 e_{2}\right)+t\left(e_{1}+e_{2}\right): 0 \leqslant t \leqslant 1\right\}$, and of the segments of the following four types:

$$
\begin{gathered}
\left\{\frac{(1-t)\left(e_{1}+e_{2}\right)}{n}+\frac{t e_{1}}{n+1}: 0 \leqslant t \leqslant 1\right\} \quad(n=1,2, \ldots) ; \\
\left\{\frac{e_{1}+(1-t) e_{2}}{n}: 0 \leqslant t \leqslant 1\right\} \quad(n=2,3, \ldots) ; \\
\left\{\frac{(1-t)\left(e_{1}+2 e_{2}\right)}{n}+\frac{2 t\left(2 e_{1}+e_{2}\right)}{4 n+3}: 0 \leqslant t \leqslant 1\right\} \quad(n=1,2, \ldots) ; \\
\left\{\frac{(1-t)\left(e_{1}+2 e_{2}\right)}{n+1}+\frac{2 t\left(2 e_{1}+e_{2}\right)}{4 n+3}: 0 \leqslant t \leqslant 1\right\} \quad(n=1,2, \ldots) .
\end{gathered}
$$

Here $e_{1}, e_{2}$ is the canonical basis in $\mathbb{R}^{2}$. By the construction of $Y$, we have $\rho_{Y}(0, E)=\infty$ for every $E \in Y \backslash\{0\}$ (see Fig. 1).

Remark 2.2. Note that if $X=\mathbb{R}^{n}$ and $U$ is a domain in $\mathbb{R}^{n}$ whose closure $Y=\operatorname{cl} U$ is a Lipschitz manifold (such that $\partial(\operatorname{cl} U)=\partial U \neq \varnothing)$, then $\rho_{\partial U, U}(x, y)=\rho_{Y}(x, y)(x, y \in \partial U)$ and $Y$ satisfies (i). Hence, this example is an important particular case of submanifolds $Y$ in a Riemannian manifold $X$ satisfying (i).

To prove our rigidity results for boundaries of submanifolds in a Riemannian manifold (see Sec. 3.), we first need to study the properties of the intrinsic geometry of these submanifolds.

One of the main results of this section is as follows:

Theorem 2.1. Let $n=2$. Then, under condition (i), the function $\rho_{Y}$ defined by (2.1) is a metric on $Y$.

Proof. It suffices to prove that $\rho_{Y}$ satisfies the triangle inequality. Let $A, O$, and $D$ be three points on the boundary of $Y$ (note that this case is basic because the other cases are simpler). Consider $\varepsilon>0$ and assume that $\gamma_{A_{\varepsilon} O_{\varepsilon}^{1}}:[0,1] \rightarrow \operatorname{Int} Y$ and $\gamma_{O_{\varepsilon}^{2} D_{\varepsilon}}:[2,3] \rightarrow \operatorname{Int} Y$ are smooth paths with the endpoints $A_{\varepsilon}=\gamma_{A_{\varepsilon} O_{\varepsilon}^{1}}(0), O_{\varepsilon}^{1}=\gamma_{A_{\varepsilon} O_{\varepsilon}^{1}}(1)$ and $D_{\varepsilon}=\gamma_{O_{\varepsilon}^{2} D_{\varepsilon}}(3)$, $O_{\varepsilon}^{2}=\gamma_{O_{\varepsilon}^{2} D_{\varepsilon}}(2)$ satisfying the conditions $\rho_{X}\left(A_{\varepsilon}, A\right) \leqslant \varepsilon, \rho_{X}\left(D_{\varepsilon}, D\right) \leqslant \varepsilon, \rho_{X}\left(O_{\varepsilon}^{j}, O\right) \leqslant \varepsilon(j=$ $1 ; 2),\left|l\left(\gamma_{A_{\varepsilon} O_{\varepsilon}^{1}}\right)-\rho_{Y}(A, O)\right| \leqslant \varepsilon$, and $\left|l\left(\gamma_{O_{\varepsilon}^{2} D_{\varepsilon}}\right)-\rho_{Y}(O, D)\right| \leqslant \varepsilon$. Let $(U, h)$ be a chart of the manifold $X$ such that $U$ is an open neighborhood of the point $O$ in $X, h(U)$ is the unit disk $B(0,1)=\left\{\left(x_{1}, x_{2}\right) \in \mathbb{R}^{2}: x_{1}^{2}+x_{2}^{2}<1\right\}$ in $\mathbb{R}^{2}$, and $h(O)=0\left(0=(0,0)\right.$ is the origin in $\left.\mathbb{R}^{2}\right) ;$ moreover $h: U \rightarrow h(U)$ is a diffeomorphism having the following property: there exists a chart $(Z, \psi)$ of $Y$ with $\psi(O)=0, A, D \in U \backslash \mathrm{cl}_{X} Z\left(\mathrm{cl}_{X} Z\right.$ is the closure of $Z$ in the space $\left.(X, g)\right)$ and $Z=\widetilde{U} \cap Y$ is the intersection of an open neighborhood $\widetilde{U}(\subset U)$ of $O$ in $X$ and $Y$ whose image $\psi(Z)$ under $\psi$ is the half-disk $B_{+}(0,1)=\left\{\left(x_{1}, x_{2}\right) \in B(0,1): x_{1} \geqslant 0\right\}$. Suppose that $\sigma_{r}$ is an arc of the circle $\partial B(0, r)$ which is a connected component of the set $V \cap \partial B(0, r)$, where 


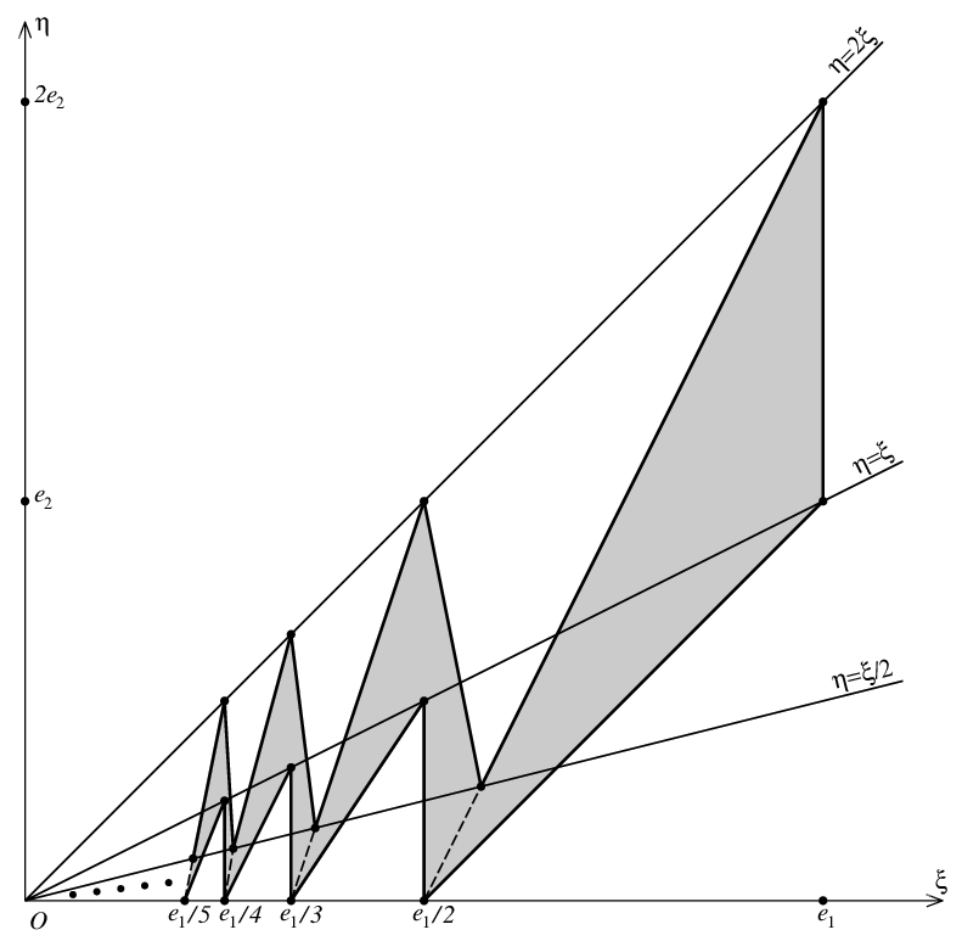

Fig. 1. An example of 2-dimensional compact connected $C^{0}$-submanifold with nonempty boundary which does not satisfy condition (i)

$V=h(Z)$ and $0<r<r^{*}=\min \left\{\left|h\left(\psi^{-1}\left(x_{1}, x_{2}\right)\right)\right|: x_{1}^{2}+x_{2}^{2}=1 / 4, x_{1} \geqslant 0\right\}$. Among these components, there is at least one (preserve the notation $\sigma_{r}$ for it) whose ends belong to the sets $h\left(\psi^{-1}\left(\left\{-t e_{2}: 0<t<1\right\}\right)\right)$ and $h\left(\psi^{-1}\left(\left\{t e_{2}: 0<t<1\right\}\right)\right)$ respectively. Otherwise, the closure of the connected component of the set $V \cap B(0, r)$ whose boundary contains the origin would contain a point belonging to the arc $\left\{e^{i \theta} / 2:|\theta| \leqslant \pi / 2\right\}$ (here we make use of the complex notation $z=r e^{i \theta}$ for points $\left.z \in \mathbb{R}^{2}(=\mathbb{C})\right)$. But this is impossible. Therefore, the above-mentioned arc $\sigma_{r}$ exists.

It is easy to check that if $\varepsilon$ is sufficiently small then the images of the paths $h \circ \gamma_{A_{\varepsilon} O_{\varepsilon}^{1}}$ and $h \circ \gamma_{O_{\varepsilon}^{2} D_{\varepsilon}}$ also intersect the arc $\sigma_{r}$, i.e., there are $\left.t_{1} \in\right] 0,1\left[, t_{2} \in\right] 2,3\left[\operatorname{such}\right.$ that $\gamma_{A_{\varepsilon} O_{\varepsilon}^{1}}\left(t_{1}\right)=x^{1^{\varepsilon}} \in Z$, $\gamma_{O_{\varepsilon}^{2} D_{\varepsilon}}\left(t_{2}\right)=x^{2} \in Z$ and $h\left(x^{j}\right) \in \sigma_{r}, j=1,2$. Let $\gamma_{r}:\left[t_{1}, t_{2}\right] \rightarrow \sigma_{r}$ be a smooth parametrization of the corresponding subarc of $\sigma_{r}$, i.e., $\gamma_{r}\left(t_{j}\right)=h\left(x^{j}\right), j=1,2$. Now we can define a mapping $\widetilde{\gamma}_{\varepsilon}:[0,3] \rightarrow$ Int $Y$ by setting

$$
\widetilde{\gamma}_{\varepsilon}(t)=\left\{\begin{array}{cc}
\gamma_{A_{\varepsilon} O_{\varepsilon}^{1}}(t), & t \in\left[0, t_{1}\right] \\
h^{-1}\left(\gamma_{r}(t)\right), & t \in] t_{1}, t_{2}[ \\
\gamma_{O_{\varepsilon}^{2} D_{\varepsilon}}(t), & t \in\left[t_{2}, 3\right]
\end{array}\right.
$$

By construction, $\widetilde{\gamma}_{\varepsilon}$ is a piecewise smooth path joining the points $A_{\varepsilon}=\widetilde{\gamma}_{\varepsilon}(0), D_{\varepsilon}=\widetilde{\gamma}_{\varepsilon}(3)$ in Int $Y$; moreover,

$$
l\left(\widetilde{\gamma}_{\varepsilon}\right) \leqslant l\left(\gamma_{A_{\varepsilon} O_{\varepsilon}^{1}}\right)+l\left(\gamma_{O_{\varepsilon}^{2} D_{\varepsilon}}\right)+l\left(h^{-1}\left(\sigma_{r}\right)\right) .
$$

By an appropriate choice of $\varepsilon>0$, we can make $r>0$ arbitrarily small, and since a piecewise smooth path can be approximated by smooth paths, we have $\rho_{Y}(A, D) \leqslant \rho_{Y}(A, O)+\rho_{Y}(O, D)$. 
In connection with Theorem 2.1, there appears a natural question: Are there analogs of this theorem for $n \geqslant 3$ ? The following Theorem 2.2 answers this question in the negative:

Theorem 2.2. If $n \geqslant 3$ then there exists an $n$-dimensional compact connected $C^{0}$-manifold $Y \subset \mathbb{R}^{n}$ with nonempty boundary $\partial Y$ such that condition (i) (where now $X=\mathbb{R}^{n}$ ) is fulfilled for $Y$ but the function $\rho_{Y}$ in this condition is not a metric on $Y$.

Proof. It suffices to consider the case of $n=3$. Suppose that $A, O, D$ are points in $\mathbb{R}^{3}, O$ is the origin in $\mathbb{R}^{3},|A|=|D|=1$, and the angle between the segments $O A$ and $O D$ is equal to $\frac{\pi}{6}$.

The manifold $Y$ will be constructed so that $O \in \partial Y$, and $] O, A] \subset \operatorname{Int} Y,] O, D] \subset \operatorname{Int} Y$. Under these conditions, $\rho_{Y}(O, A)=\rho_{Y}(O, D)=1$. However, the boundary of $Y$ will create "obstacles" between $A$ and $D$ such that the length of any curve joining $A$ and $D$ in $\operatorname{Int} Y$ will be greater than $\frac{12}{5}$ (this means the violation of the triangle inequality for $\rho_{Y}$ ).

Consider a countable collection of mutually disjoint segments $\left\{I_{j}^{k}\right\}_{j \in \mathbb{N}, k=1, \ldots, k_{j}}$ lying in the interior of the triangle $6 \triangle A O D$ (which is obtained from the original triangle $\triangle A O D$ by dilation with coefficient 6 ) with the following properties:

$(*)$ every segment $I_{j}^{k}=\left[x_{j}^{k}, y_{j}^{k}\right]$ lies on a ray starting at the origin, $y_{j}^{k}=11 x_{j}^{k}$, and $\left|x_{j}^{k}\right|=2^{-j}$;

(**) any curve $\gamma$ with ends $A, D$ whose interior points lie in the interior of the triangle $4 \triangle A O D$ and belong to no segment $I_{j}^{k}$, satisfies the estimate $l(\gamma) \geqslant 6$.

The existence of such a family of segments is certain: the segments of the family must be situated chequerwise so that any curve disjoint from them be sawtooth, with the total length of its "teeth" greater than 6 (it can clearly be made greater than any prescribed positive number). However, below we exactly describe the construction.

It is easy to include the above-indicated family of segments in the boundary $\partial Y$ of $Y$. Thus, it creates a desired "obstacle" to joining $A$ and $D$ in the plane of $\triangle A O D$. But it makes no obstacle to joining $A$ and $D$ in the space. The simplest way to create such a space obstacle is as follows: Rotate each segment $I_{j}^{k}$ along a spiral around the axis $O A$. Make the number of coils so large that the length of this spiral be large and its pitch (i.e., the distance between the origin and the end of a coil) be sufficiently small. Then the set $S_{j}^{k}$ obtained as the result of the rotation of the segment $I_{j}^{k}$ is diffeomorphic to a plane rectangle, and it lies in a small neighborhood of the cone of revolution with axis $A O$ containing the segment $I_{j}^{k}$. The last circumstance guarantees that the sets $S_{j}^{k}$ are disjoint as before, and so (as above) it is easy to include them in the boundary $\partial Y$ but, due to the properties of the $I_{j}^{k}$ 's and a large number of coils of the spirals $S_{j}^{k}$, any curve joining $A, D$ and disjoint from each $S_{j}^{k}$ has length $\geqslant \frac{12}{5}$.

We turn to an exact description of the constructions used. First describe the construction of the family of segments $I_{j}^{k}$. They are chosen on the basis of the following observation:

Let $\gamma:[0,1] \rightarrow 4 \triangle A O D$ be any curve with ends $\gamma(0)=A, \gamma(1)=D$ whose interior points lie in the interior of the triangle $4 \Delta A O D$. For $j \in \mathbb{N}$, put $R_{j}=\left\{x \in 4 \Delta A O D:|x| \in\left[8 \cdot 2^{-j}, 4 \cdot 2^{-j}\right]\right\}$. It is clear that

$$
4 \triangle A O D \backslash\{O\}=\cup_{j \in \mathbb{N}} R_{j} .
$$

Introduce the polar system of coordinates on the plane of the triangle $\triangle A O D$ with center $O$ such that the coordinates of the points $A, D$ are $r=1, \varphi=0$ and $r=1, \varphi=\frac{\pi}{6}$, respectively. Given a point $x \in 6 \triangle A O D$, let $\varphi_{x}$ be the angular coordinate of $x$ in $\left[0, \frac{\pi}{6}\right]$. Let $\Phi_{j}=\left\{\varphi_{\gamma(t)}: \gamma(t) \in R_{j}\right\}$. Obviously, there is $j_{0} \in \mathbb{N}$ such that

$$
\mathcal{H}^{1}\left(\Phi_{j_{0}}\right) \geqslant 2^{-j_{0}} \frac{\pi}{6}
$$

where $\mathcal{H}^{1}$ is the Hausdorff 1 -measure. This means that, while in the layer $R_{j_{0}}$, the curve $\gamma$ covers the angular distance $\geqslant 2^{-j_{0}} \frac{\pi}{6}$. The segments $I_{j}^{k}$ must be chosen such that $(2.2)$ together with 
the condition

$$
\gamma(t) \cap I_{j}^{k}=\varnothing \quad \forall t \in[0,1], \forall j \in \mathbb{N}, \forall k \in\left\{1, \ldots, k_{j}\right\}
$$

give the desired estimate $l(\gamma) \geqslant 6$. To this end, it suffices to take $k_{j}=\left[(2 \pi)^{j}\right]$ (the integral part of $\left.(2 \pi)^{j}\right)$ and

$$
I_{j}^{k}=\left\{x \in 6 \Delta A O D: \varphi_{x}=k(2 \pi)^{-j} \frac{\pi}{6},|x| \in\left[11 \cdot 2^{-j}, 2^{-j}\right]\right\}
$$

$k=1, \ldots, k_{j}$. Indeed, under this choice of the $I_{j}^{k}$ 's, estimate $(2.2)$ implies that $\gamma$ must intersect at least $(2 \pi)^{j_{0}} 2^{-j_{0}}=\pi^{j_{0}}>3^{j_{0}}$ of the figures

$$
U_{k}=\left\{x \in R_{j_{0}}: \varphi_{x} \in\left(k(2 \pi)^{-j_{0}} \frac{\pi}{6},(k+1)(2 \pi)^{-j_{0}} \frac{\pi}{6}\right)\right\} .
$$

Since these figures are separated by the segments $I_{j_{0}}^{k}$ in the layer $R_{j_{0}}$, the curve $\gamma$ must be disjoint from them each time in passing from one figure to another. The number of these passages must be at least $3^{j_{0}}-1$, and a fragment of $\gamma$ of length at least $2 \cdot 3 \cdot 2^{-j_{0}}$ is required for each passage (because the ends of the segments $I_{j_{0}}^{k}$ go beyond the boundary of the layer $R_{j_{0}}$ containing the figures $U_{k}$ at distance $\left.3 \cdot 2^{-j_{0}}\right)$. Thus, for all these passages, a section of $\gamma$ is spent of length at least

$$
6 \cdot 2^{-j_{0}}\left(3^{j_{0}}-1\right) \geqslant 6
$$

Hence, the construction of the segments $I_{j}^{k}$ satisfying $(*)-(* *)$ is finished.

Let us now describe the construction of the above-mentioned space spirals.

For $x \in \mathbb{R}^{3}$, denote by $\Pi_{x}$ the plane that passes through $x$ and is perpendicular to the segment $O A$. On $\Pi_{x_{j}^{k}}$, consider the polar coordinates $(\rho, \psi)$ with origin at the point of intersection of $\Pi_{x_{j}^{k}}$ and $[O, A]$ (in this system, the point $x_{j}^{k}$ has coordinates $\rho=\rho_{j}^{k}, \psi=0$ ). Suppose that a point $x(\psi) \in \Pi_{x_{j}^{k}}$ moves along an Archimedean spiral, namely, the polar coordinates of the point $x(\psi)$ are $\rho(\psi)=\rho_{j}^{k}-\varepsilon_{j} \psi, \psi \in\left[0,2 \pi M_{j}\right]$, where $\varepsilon_{j}$ is a small parameter to be specified below, and $M_{j} \in \mathbb{N}$ is chosen so large that the length of any curve passing between all coils of the spiral is at least 10 .

Describe the choice of $M_{j}$ more exactly. To this end, consider the points $x(2 \pi), x\left(2 \pi\left(M_{j}-1\right)\right)$, $x\left(2 \pi M_{j}\right)$, which are the ends of the first, penultimate, and last coils of the spiral respectively (with $x(0)=x_{j}^{k}$ taken as the starting point of the spiral). Then $M_{j}$ is chosen so large that the following condition hold:

$\left(*_{1}\right)$ The length of any curve on the plane $\Pi_{x_{j}^{k}}$, joining the segments $\left[x_{j}^{k}, x(2 \pi)\right]$ and $\left[x\left(2 \pi\left(M_{j}-1\right)\right), x\left(2 \pi M_{j}\right)\right]$ and disjoint from the spiral $\left\{x(\psi): \psi \in\left[0,2 \pi M_{j}\right]\right\}$, is at least 10 .

Figuratively speaking, the constructed spiral bounds a "labyrinth", the mentioned segments are the entrance to and the exit from this labyrinth, and thus any path through the labyrinth has length $\geqslant 10$.

Now, start rotating the entire segment $I_{j}^{k}$ in space along the above-mentioned spiral, i.e., assume that $I_{j}^{k}(\psi)=\{y=\lambda x(\psi): \lambda \in[1,11]\}$. Thus, the segment $I_{j}^{k}(\psi)$ lies on the ray joining $O$ with $x(\psi)$ and has the same length as the original segment $I_{j}^{k}=I_{j}^{k}(0)$. Define the surface $S_{j}^{k}=\cup_{\psi \in\left[0,2 \pi M_{j}\right]} I_{j}^{k}(\psi)$. This surface is diffeomorphic to a plane rectangle (strip). Taking $\varepsilon_{j}>0$ sufficiently small, we may assume without loss of generality that $2 \pi M_{j} \varepsilon_{j}$ is substantially less than $\rho_{j}^{k}$; moreover, that the surfaces $S_{j}^{k}$ are mutually disjoint (obviously, the smallness of $\varepsilon_{j}$ does not affect property $\left(*_{1}\right)$ which in fact depends on $\left.M_{j}\right)$.

Denote by $y(\psi)=11 x(\psi)$ the second end of the segment $I_{j}^{k}(\psi)$. Consider the trapezium $P_{j}^{k}$ with vertices $y_{j}^{k}, x_{j}^{k}, x\left(2 \pi M_{j}\right), y\left(2 \pi M_{j}\right)$ and sides $I_{j}^{k}, I_{j}^{k}\left(2 \pi M_{j}\right),\left[x_{j}^{k}, x\left(2 \pi M_{j}\right)\right]$, and $\left[y_{j}^{k}, y\left(2 \pi M_{j}\right)\right]$ (the last two sides are parallel since they are perpendicular to the segment $A O$ ). By construction, $P_{j}^{k}$ lies on the plane $A O D$; moreover, taking $\varepsilon_{j}$ sufficiently small, we can obtain the situation where the trapeziums $P_{j}^{k}$ are mutually disjoint (since $P_{j}^{k} \rightarrow I_{j}^{k}$ under fixed $M_{j}$ and $\varepsilon_{j} \rightarrow 0$ ). 
Take an arbitrary triangle whose vertices lie on $P_{j}^{k}$ and such that one of these vertices is also a vertex at an acute angle in $P_{j}^{k}$. By construction, this acute angle is at least $\frac{\pi}{2}-\angle A O D=\frac{\pi}{3}$. Therefore, the ratio of the side of the triangle lying inside the trapezium $P_{j}^{k}$ to the sum of the other two sides (lying on the corresponding sides of $P_{j}^{k}$ ) is at least $\frac{1}{2} \sin \frac{\pi}{3}>\frac{2}{5}$. If we consider the same ratio for the case of a triangle with a vertex at an obtuse angle of $P_{j}^{k}$ then it is greater than $\frac{1}{2}$. Thus, we have the following property:

$\left(*_{2}\right)$ For arbitrary triangle whose vertices lie on the trapezium $P_{j}^{k}$ and one of these vertices is also a vertex in $P_{j}^{k}$, the sum of lengths of the sides situated on the corresponding sides of $P_{j}^{k}$ is less than $\frac{5}{2}$ of the length of the third side (lying inside $P_{j}^{k}$ ).

Let a point $x$ lie inside the cone $K$ formed by the rotation of the angle $\angle A O D$ around the ray $O A$. Denote by Proj $\mathrm{jot}_{\mathrm{x}} \mathrm{x}$ the point of the angle $\angle A O D$ which is the image of $x$ under this rotation. Finally, let $K_{4 \triangle A O D}$ stand for the corresponding truncated cone obtained by the rotation of the triangle $4 \triangle A O D$, i.e., $K_{4 \triangle A O D}=\left\{x \in K:\right.$ Proj $\left._{\text {rot }} \mathrm{x} \in 4 \Delta \mathrm{AOD}\right\}$.

The key ingredient in the proof of our theorem is the following assertion:

$\left(*_{3}\right)$ For arbitrary space curve $\gamma$ of length less than 10 joining the points $A$ and $D$, contained in the truncated cone $K_{4 \triangle A O D} \backslash\{O\}$, and disjoint from each strip $S_{j}^{k}$, there exists a plane curve $\tilde{\gamma}$ contained in the triangle $4 \triangle A O D \backslash\{O\}$, that joins $A$ and $D$, is disjoint from all segments $I_{j}^{k}$ and such that the length of $\tilde{\gamma}$ is less than $\frac{5}{2}$ of the length of Projrot $\gamma$.

Prove $\left(*_{3}\right)$. Suppose that its hypotheses are fulfilled. In particular, assume that the inclusion Projrot $\gamma \subset 4 \Delta \mathrm{AOD} \backslash\{\mathrm{O}\}$ holds. We need to modify Proj Prot $\gamma$ so that the new curve be contained in the same set but be disjoint from each of the $I_{j}^{k}$ 's. The construction splits into several steps.

Step 1. If Projrot $\gamma$ intersects a segment $I_{j}^{k}$ then it necessarily intersects also at least one of the shorter sides of $P_{j}^{k}$.

Recall that, by construction, $P_{j}^{k}=$ Projrot $_{\mathrm{j}} \mathrm{S}_{\mathrm{j}}^{\mathrm{k}}$; moreover, $\gamma$ intersects no spiral strip $S_{j}^{k}$. If Proj $_{\text {rot }} \gamma$ intersected $P_{j}^{k}$ without intersecting its shorter sides then $\gamma$ would pass through all coils of the corresponding spiral. Then, by $\left(*_{1}\right)$, the length of the corresponding fragment of $\gamma$ would be $\geqslant 10$ in contradiction to our assumptions. Thus, the assertion of step 1 is proved.

Step 2. Denote by $\gamma_{P_{j}^{k}}$ the fragment of the plane curve Projrot $\gamma$ beginning at the first point of its entrance into the trapezium $P_{j}^{k}$ to the point of its exit from $P_{j}^{k}$ (i.e., to its last intersection point with $P_{j}^{k}$ ). Then this fragment $\gamma_{P_{j}^{k}}$ can be deformed without changing the first and the last points so that the corresponding fragment of the new curve lie entirely on the union of the sides of $P_{j}^{k}$; moreover, its length is less than $\frac{5}{2}$ of the length of $\gamma_{P_{j}^{k}}$.

The assertion of step 2 immediately follows from the assertions of step 1 and $\left(*_{2}\right)$.

The assertion of step 2 in turn directly implies the desired assertion $\left(*_{3}\right)$. The proof of $\left(*_{3}\right)$ is finished.

Now, we are ready to pass to the final part of the proof of Theorem 2.2.

$\left(*_{4}\right)$ The length of any space curve $\gamma \subset \mathbb{R}^{3} \backslash\{O\}$ joining $A$ and $D$ and disjoint from each strip $S_{j}^{k}$ is at least $\frac{12}{5}$.

Prove the last assertion. Without loss of generality, we may also assume that all interior points of $\gamma$ are inside the cone $K$ (otherwise the initial curve can be modified without any increase of its length so that assumptions of $\left(*_{4}\right)$ are still fulfilled and the modified curve lies in $K$ ). If $\gamma$ is not included in the truncated cone $K_{4 \triangle A O D} \backslash\{O\}$ then Proj $j_{\text {rot }} \gamma$ intersects the segment $[4 A, 4 D]$; consequently, the length of $\gamma$ is at least $2(4 \sin \angle O A D-1)=2\left(4 \sin \frac{\pi}{3}-1\right)=2(2 \sqrt{3}-1)>4$, and the desired estimate is fulfilled. Similarly, if the length of $\gamma$ is at least 10 then the desired 
estimate is fulfilled automatically, and there is nothing to prove. Hence, we may further assume without loss of generality that $\gamma$ is included in the truncated cone $K_{4 \triangle A O D} \backslash\{O\}$ and its length is less than 10. Then, by $\left(*_{3}\right)$, there is a plane curve $\tilde{\gamma}$ contained in the triangle $4 \triangle A O D \backslash\{O\}$, joining the points $A$ and $D$, disjoint from each segment $I_{j}^{k}$, and such that the length of $\tilde{\gamma}$ is at most $\frac{5}{2}$ of the length of Projrot $\gamma$. By property $(* *)$ of the family of segments $I_{j}^{k}$, the length of $\tilde{\gamma}$ is at least 6 . Consequently, the length of $\operatorname{Proj}_{\text {rot }} \gamma$ is at least $\frac{12}{5}$, which implies the desired estimate. Assertion $\left(*_{4}\right)$ is proved.

The just-proven property $\left(*_{4}\right)$ of the constructed objects implies Theorem 2.2. Indeed, since the strips $S_{j}^{k}$ are mutually disjoint and, outside every neighborhood of the origin $O$, there are only finitely many of these strips, it is easy to construct a $C^{0}$-manifold $Y \subset \mathbb{R}^{3}$ that is homeomorphic to a closed ball (i.e., $\partial Y$ is homeomorphic to a two-dimensional sphere) and has the following properties:

(I) $O \in \partial Y,[A, O[\cup[D, O[\subset \operatorname{Int} Y$;

(II) for every point $y \in(\partial Y) \backslash\{O\}$, there exists a neighborhood $U(y)$ such that $U(y) \cap \partial Y$ is $C^{1}$-diffeomorphic to the plane square $[0,1]^{2}$;

(III) $S_{j}^{k} \subset \partial Y$ for all $j \in \mathbb{N}, k=1, \ldots, k_{j}$.

The construction of $Y$ with properties (I)-(III) can be carried out, for example, as follows: As the surface of the zeroth step, take a sphere containing $O$ and such that $A$ and $D$ are inside the sphere. At the $j$ th step, a small neighborhood of the point $O$ of our surface is smoothly deformed so that the modified surface is still smooth, homeomorphic to a sphere, and contains all strips $S_{j}^{k}, k=1, \ldots, k_{j}$. Besides, we make sure that, at the each step, the so-obtained surface be disjoint from the half-intervals $\left[A, O\left[\right.\right.$ and $\left[D, O\left[\right.\right.$, and, as above, contain all strips $S_{i}^{k}, i \leqslant j$, already included therein. Since the neighborhood we are deforming contracts to the point $O$ as $j \rightarrow \infty$, the so-constructed sequence of surfaces converges (for example, in the Hausdorff metric) to a limit surface which is the boundary of a $C^{0}$-manifold $Y$ with properties (I)-(III).

Property (I) guarantees that $\rho_{Y}(A, O)=\rho_{Y}(A, D)=1$ and $\rho_{Y}(O, x) \leqslant 1+\rho_{Y}(A, x)$ for all $x \in Y$. Property (II) implies the estimate $\rho_{Y}(x, y)<\infty$ for all $x, y \in Y \backslash\{O\}$, which, granted the previous estimate, yields $\rho_{Y}(x, y)<\infty$ for all $x, y \in Y$. However, property (III) and the assertion $\left(*_{4}\right)$ imply that $\rho_{Y}(A, D) \geqslant \frac{12}{5}>2=\rho_{Y}(A, O)+\rho_{Y}(A, D)$. Theorem 2.2 is proved.

If $\rho_{Y}$ is a metric (the dimension $n(\geqslant 2)$ is arbitrary) then the question of the existence of geodesics is solved in the following assertion, which implies that $\rho_{Y}$ is the intrinsic metric (see, for example, $\S 6$ in [11]).

Theorem 2.3. Assume that $\rho_{Y}$ is a finite function and is a metric on $Y$. Then any two points $x, y \in Y$ can be joined in $Y$ by a shortest curve $\gamma:[0, L] \rightarrow Y$ in the metric $\rho_{Y} ;$ i.e., $\gamma(0)=x$, $\gamma(L)=y$, and

$$
\rho_{Y}(\gamma(s), \gamma(t))=t-s, \quad \forall s, t \in[0, L], \quad s<t .
$$

Proof. Fix a pair of distinct points $x, y \in Y$ and put $L=\rho_{Y}(x, y)$. Now, take a sequence of paths $\gamma_{j}:[0, L] \rightarrow \operatorname{Int} Y$ such that $\gamma_{j}(0)=x_{j}, \gamma_{j}(L)=y_{j}, x_{j} \rightarrow x, y_{j} \rightarrow y$, and $l\left(\gamma_{j}\right) \rightarrow L$ as $j \rightarrow \infty$. Without loss of generality, we may also assume that the parametrizations of the curves $\gamma_{j}$ are their natural parametrizations up to a factor (tending to 1 ) and the mappings $\gamma_{j}$ converge uniformly to a mapping $\gamma:[0, L] \rightarrow Y$ with $\gamma(0)=x, \gamma(L)=y$. By these assumptions,

$$
\lim _{j \rightarrow \infty} l\left(\left.\gamma_{j}\right|_{[s, t]}\right)=t-s \quad \forall s, t \in[0, L], \quad s<t .
$$

Take an arbitrary pair of numbers $s, t \in[0, L], s<t$. By construction, we have the convergence $\gamma_{j}(s) \in \operatorname{Int} Y \rightarrow \gamma(s), \gamma_{j}(t) \in \operatorname{Int} Y \rightarrow \gamma(t)$ as $j \rightarrow \infty$. From here and the definition of the 
metric $\rho_{Y}(\cdot, \cdot)$ it follows that $\rho_{Y}(\gamma(s), \gamma(t)) \leqslant \lim _{j \rightarrow \infty} l\left(\left.\gamma_{j}\right|_{[s, t]}\right)$. By $(2.4)$,

$$
\rho_{Y}(\gamma(s), \gamma(t)) \leqslant t-s \quad \forall s, t \in[0, L], s<t .
$$

Prove that (2.5) is indeed an equality. Assume that $\rho_{Y}\left(\gamma\left(s^{\prime}\right), \gamma\left(t^{\prime}\right)\right)<t^{\prime}-s^{\prime}$ for some $s^{\prime}, t^{\prime} \in[0, L]$, $s^{\prime}<t^{\prime}$. Then, applying the triangle inequality and then (2.5), we infer

$$
\rho_{Y}(x, y) \leqslant \rho_{Y}\left(x, \gamma\left(s^{\prime}\right)\right)+\rho_{Y}\left(\gamma\left(s^{\prime}\right), \gamma\left(t^{\prime}\right)\right)+\rho_{Y}\left(\gamma\left(t^{\prime}\right), y\right)<s^{\prime}+\left(t^{\prime}-s^{\prime}\right)+\left(L-t^{\prime}\right)=L,
$$

which contradicts the initial equality $\rho_{Y}(x, y)=L$. The so-obtained contradiction completes the proof of identity (2.3).

Remark 2.3. Identity (2.3) means that the curve of Theorem 2.3 is a geodesic in the metric $\rho_{Y}$, i.e., the length of its fragment between points $\gamma(s), \gamma(t)$ calculated in $\rho_{Y}$ is equal to $\rho_{Y}(\gamma(s), \gamma(t))=t-s$. Nevertheless, if we compute the length of the above-mentioned fragment of the curve in the initial Riemannian metric then this length need not coincide with $t-s$; only the easily verifiable estimate $l\left(\left.\gamma\right|_{[s, t]}\right) \leqslant t-s$ holds (see (2.4)). In the general case, the equality $l\left(\left.\gamma\right|_{[s, t]}\right)=t-s$ can only be guaranteed if $n=2$ (if $n \geqslant 3$ then the corresponding counterexample is constructed by analogy with the counterexample in the proof of Theorem 2.2, see above). In particular, though, by Theorem 2.3, the metric $\rho_{Y}$ is always intrinsic in the sense of the definitions in $[11, \S 6]$, the space $\left(Y, \rho_{Y}\right)$ may fail to be a space with intrinsic metric in the sense of [ibid].

\section{Rigidity theorems for the boundaries of submanifolds in Riemannian manifolds}

As in Sec. 2., let $(X, g)$ be an $n$-dimensional smooth connected Riemannian manifold without boundary and let $\rho_{X}$ be its intrinsic metric (i.e., let $\rho_{X}(x, y)$ be the infimum of the lengths $l\left(\gamma_{x, y, X}\right)$ of smooth paths $\gamma_{x, y, X}:[0,1] \rightarrow X$ joining points $x$ and $y$ in a manifold $\left.X\right)$.

Assume that $Y$ is an $n$-dimensional compact connected $C^{0}$-submanifold $Y \subset X$ with nonempty boundary $\partial Y$ satisfying condition (i) in Sec. 2., moreover, $\rho_{Y}$ is a metric on $Y$. Then $Y$ is called strictly convex in the metric $\rho_{Y}$ if, for any $\alpha, \beta \in Y$, any shortest path $\gamma=\gamma_{\alpha, \beta, Y}:[0,1] \rightarrow Y$ between $\alpha$ and $\beta$ (in the metric $\rho_{Y}$ ) satisfies $\gamma(] 0,1[) \subset \operatorname{Int} Y$.

Theorem 3.1. Let $n=2$. Assume that condition (i) holds for a 2-dimensional compact connected $C^{0}$-submanifold $Y_{1}$ with nonempty boundary $\partial Y_{1}$ of a 2-dimensional smooth connected Riemannian manifold $X$ without boundary which is strictly convex in the metric $\rho_{Y_{1}}$. Suppose that $Y_{2} \subset X$ is also a 2-dimensional compact connected $C^{0}$-submanifold of $X$ with $\partial Y_{2} \neq \varnothing$ satisfying (i); moreover, $\partial Y_{1}$ and $\partial Y_{2}$ are isometric in the metrics $\rho_{Y_{j}}$, for $j=1,2$. Then, $Y_{2}$ is strictly convex with respect to $\rho_{Y_{2}}$.

Proof. Suppose that, for points $x, y \in Y_{2}$, there exists a shortest path $\gamma_{x, y, Y_{2}}:[0,1] \rightarrow Y_{2}$ in the metric $\rho_{Y_{2}}$ joining $x$ and $y$ and such that $\left\{\gamma_{x, y, Y_{2}}(] 0,1[)\right\} \cap \partial Y_{2} \neq \varnothing$, i.e., $x^{\prime}=\gamma_{x, y, Y_{2}}\left(t^{\prime}\right) \in$ $\left\{\gamma_{x, y, Y_{2}}(] 0,1[) \cap \partial Y_{2}\right\}$ for a point $\left.t^{\prime} \in\right] 0,1\left[\right.$. By Theorem 2.3 and the fact that $Y_{2}$ is a 2-dimensional compact connected $C^{0}$-submanifold in $X$, for a sufficiently small neighborhood of $x^{\prime}$ in $Y_{2}$, we can find points $x_{0}, y_{0} \in \partial Y_{2}$ and a shortest path $\gamma_{x_{0}, y_{0}, Y_{2}}:[0,1] \rightarrow Y_{2}$ between $x_{0}$ and $y_{0}$ in the same metric satisfying the condition $x^{\prime} \in\left\{\gamma_{x_{0}, y_{0}, Y_{2}}(] 0,1[) \cap \partial Y_{2}\right\}$. Further, we will suppose that $x=x_{0}$ and $y=y_{0}$.

Now, assume that $f: \partial Y_{1} \rightarrow \partial Y_{2}$ is an isometry of $\partial Y_{1}$ and $\partial Y_{2}$ in the metrics $\rho_{Y_{1}}$ and $\rho_{Y_{2}}$ of the boundaries $\partial Y_{1}$ and $\partial Y_{2}$ of the submanifolds $Y_{1}$ and $Y_{2}$ of $X$. From Theorem 2.3, we have

$$
\rho_{Y_{2}}\left(x, x^{\prime}\right)+\rho_{Y_{2}}\left(x^{\prime}, y\right)=l_{1}+l_{2}=l=\rho_{Y_{2}}(x, y) .
$$


Since $f$ is an isometry,

$$
\rho_{Y_{1}}\left(f^{-1}(x), f^{-1}\left(x^{\prime}\right)\right)+\rho_{Y_{1}}\left(f^{-1}\left(x^{\prime}\right), f^{-1}(y)\right)=\rho_{Y_{2}}\left(x, x^{\prime}\right)+\rho_{Y_{2}}\left(x^{\prime}, y\right) .
$$

Next, consider shortest paths $\gamma_{f^{-1}(x), f^{-1}\left(x^{\prime}\right), Y_{1}}:[0,1 / 2] \rightarrow Y_{1}$ and $\gamma_{f^{-1}\left(x^{\prime}\right), f^{-1}(y), Y_{1}}:[1 / 2,1] \rightarrow$ $Y_{1}$ in $\rho_{Y_{1}}$ between (respectively) $f^{-1}(x)$ and $f^{-1}\left(x^{\prime}\right)$ and $f^{-1}\left(x^{\prime}\right)$ and $f^{-1}(y)$, and then construct a path $\gamma:[0,1] \rightarrow Y_{1}$ by setting $\gamma(t)=\gamma_{f^{-1}(x), f^{-1}\left(x^{\prime}\right), Y_{1}}(t)$ if $0 \leqslant t<1 / 2$ and $=\gamma_{f^{-1}\left(x^{\prime}\right), f^{-1}(y), Y_{1}}(t)$ for $1 / 2 \leqslant t \leqslant 1$. Let $l_{Y_{1}}(\delta)$ be the length of a path $\delta:[0,1] \rightarrow Y_{1}$ in the metric $\rho_{Y_{1}}$. Since $\rho_{Y_{1}}$ is a metric on $Y_{1}$, it is not difficult to show that

$$
l_{Y_{1}}(\gamma) \leqslant l_{Y_{1}}\left(\gamma_{f^{-1}(x), f^{-1}\left(x^{\prime}\right), Y_{1}}\right)+l_{Y_{1}}\left(\gamma_{f^{-1}\left(x^{\prime}\right), f^{-1}(y), Y_{1}}\right)=l_{1}+l_{2}
$$

Hence $\gamma$ is a shortest path in $\rho_{Y_{1}}$ joining $f^{-1}(x)$ and $f^{-1}(y)$ in $Y_{1}$. This contradicts the strict convexity of $Y_{1}$. The theorem is proved.

Corollary 3.1. Suppose that the conditions of Theorem 3.1 hold and the manifold $X$ has the following property: $\rho_{X}(x, y)=\rho_{Y}(x, y)$ for any two points $x$ and $y$ from every 2-dimensional compact connected $C^{0}$-submanifold $Y \subset X$ with $\partial Y \neq \varnothing$ satisfying condition (i) and strictly convex with respect to the metric $\rho_{Y}$. Then, $\partial Y_{1}$ and $\partial Y_{2}$ are isometric in the metric $\rho_{X}$ on the ambient manifold $X$.

Remark 3.1. The condition imposed on the manifold $X$ in Corollary 3.1 can be reformulated as follows: in this manifold, every 2-dimensional compact connected $C^{0}$-submanifold $Y$ with boundary satisfying condition (i) and strictly convex with respect to its intrinsic metric $\rho_{Y}$ is a convex set in the ambient space $X$ with respect to the metric $\rho_{X}$ (for the notion of a convex set in a metric space the reader is referred, for example, to [11]).

We have the following analog of Theorem 3.1 and Corollary 3.1 (combined together) for $n \geqslant 3$ :

Theorem 3.2. Let $n \geqslant 3$. Suppose that $(X, g)$ is an $n$-dimensional smooth connected Riemannian manifold without boundary and $Y_{1}$ and $Y_{2}$ are $n$-dimensional compact connected $C^{0}$ submanifolds with nonempty boundaries $\partial Y_{1}$ and $\partial Y_{2}$ in $X$ satisfying conditions (i), and

(ii) $\rho_{Y_{j}}$ is a metric on $Y_{j}(j=1,2)$,

(iii) for any two points $a, b \in Y_{j}$, there exist points $c, d \in \partial Y_{j}$ which can be joined in $Y_{j}$ by a shortest path $\gamma:[0,1] \rightarrow Y_{j}$ in the metric $\rho_{Y_{j}}$ so that $a, b \in \gamma([0,1])$.

Furthermore, assume that $Y_{1}$ is strictly convex in the metric $\rho_{Y_{1}}, X$ has the additional property that $\rho_{X}(x, y)=\rho_{Y}(x, y)$ for any two points $x$ and $y$ in every $n$-dimensional compact connected $C^{0}$-submanifold $Y \subset X$ with $\partial Y \neq \varnothing$ satisfying conditions (i)-(iii) and strictly convex with respect to $\rho_{Y}$ and the boundaries $\partial Y_{1}$ and $\partial Y_{2}$ of the submanifolds $Y_{1}$ and $Y_{2}$ are isometric with respect to the metrics $\rho_{Y_{j}}$, where $j=1,2$. Then, $\partial Y_{1}$ and $\partial Y_{2}$ are isometric with respect to $\rho_{X}$.

Remark 3.2. For a submanifold $Y$ in $X$, (i) and (ii) can be considered as conditions of generalized regularity near its boundary.

Remark 3.3. Theorem 3.1, Corollary 3.1, and Theorem 3.2 are closely related to a theorem of A.D. Aleksandrov about the rigidity of the boundary $\partial U$ of a strictly convex domain $U$ in Euclidean $n$-space $\mathbb{R}^{n}$ by the relative metric $\rho_{\partial U, U}$ on the boundary. The following is an important particular case of this theorem:

Theorem 3.3 (A. D. Aleksandrov ( [6])). Let $U_{1}$ be a strictly convex domain in $\mathbb{R}^{n}$ (i.e., for any $\alpha, \beta \in \operatorname{cl} U_{1}$ every shortest path $\gamma=\gamma_{\alpha, \beta, \operatorname{cl} U_{1}}:[0,1] \rightarrow \operatorname{cl} U_{1}$ between $\alpha$ and $\beta$ (in the metric $\left.\rho_{\mathrm{cl} U_{1}}\right)$ satisfies $\left.\gamma(] 0,1[) \subset U_{1}\right)$. Assume that $U_{2} \subset \mathbb{R}^{n}$ is any domain whose closure is a Lipschitz manifold (such that $\left.\partial\left(\mathrm{cl} U_{2}\right)=\partial U_{2} \neq \varnothing\right)$; moreover, $\partial U_{1}$ and $\partial U_{2}$ are isometric in their relative metrics $\rho_{\partial U_{1}, U_{1}}$ and $\rho_{\partial U_{2}, U_{2}}$. Then $\partial U_{1}$ and $\partial U_{2}$ are isometric in the Euclidean metric. 
We say that an $n$-dimensional compact (closed) connected $C^{0}$-submanifold $Y$ with boundary $\partial Y \neq \varnothing$ of an $n$-dimensional smooth connected (respectively, $n$-dimensional smooth complete connected) Riemannian manifold $X$ without boundary has property (o) if $\gamma_{x, y, Y}(] 0,1[) \subset$ Int $Y$ for any two points $x, y \in \partial Y$ and for every shortest path $\gamma_{x, y, Y}:[0,1] \rightarrow Y$ in the metric $\rho_{Y}$ joining these points.

Theorem 3.4. Let $n=2$. Suppose that (i) holds for a 2-dimensional compact connected $C^{0}$ submanifold $Y_{1}$ with boundary $\partial Y_{1} \neq \varnothing$ in a 2-dimensional smooth connected Riemannian manifold $X$ without boundary; moreover, $Y_{1}$ has property (o). Assume that $Y_{2} \subset X$ is a 2-dimensional compact connected $C^{0}$-submanifold with $\partial Y_{2} \neq \varnothing$ in $X$ and $\partial Y_{1}$ and $\partial Y_{2}$ are isometric in the metrics $\rho_{Y_{j}}(j=1,2)$. Then $\partial Y_{2}$ also has property $(\circ)$.

This theorem has the following generalization.

Theorem 3.5. Let $n=2$. Suppose that (i) holds for a 2-dimensional closed connected $C^{0}$ submanifold $Y_{1}$ with boundary $\partial Y_{1}(\neq \varnothing)$ in a 2-dimensional smooth complete connected Riemannian manifold $X$ without boundary satisfying (०). Assume that $Y_{2} \subset X$ is a 2-dimensional closed connected $C^{0}$-submanifold with $\partial Y_{2} \neq \varnothing$ in $X$; moreover, $\partial Y_{1}$ and $\partial Y_{2}$ are isometric in the metrics $\rho_{Y_{j}}(j=1,2)$. Then $Y_{2}$ has the property (०) as well.

Corollary 3.2 (of Theorem 3.4). Assume that the hypothesis of Theorem 3.4 hold and that the manifold $X$ has the following property: $\rho_{X}(x, y)=\rho_{Y}(x, y)$ for any two points $x$ and $y$ on the boundary $\partial Y$ of every 2-dimensional compact connected $C^{0}$-submanifold $Y \subset X$ with $\partial Y \neq \varnothing$ satisfying $(\mathrm{i})$ and $(\mathrm{o})$. Then $\partial Y_{1}$ and $\partial Y_{2}$ are isometric in the metric $\rho_{X}$ of the ambient manifold $X$.

Corollary 3.3 (of Theorem 3.5). Assume that the hypothesis of Theorem 3.5 hold and that the manifold $X$ has the following property: $\rho_{X}(x, y)=\rho_{Y}(x, y)$ for any two points $x$ and $y$ on the boundary $\partial Y$ of every 2-dimensional closed connected $C^{0}$-submanifold $Y \subset X$ with $\partial Y \neq \varnothing$ satisfying (i) and (o). Then $\partial Y_{1}$ and $\partial Y_{2}$ are isometric with respect to $\rho_{X}$.

Theorem 3.6. Let $n \geqslant 3$. Suppose that $(X, g)$ is an $n$-dimensional smooth connected Riemannian manifold whithout boundary and $Y_{1}$ and $Y_{2}$ are $n$-dimensional compact connected $C^{0}$ submanifolds with nonempty boundaries $\partial Y_{1}$ and $\partial Y_{2}$ in $X$ satisfying conditions (i) and (ii) (in Theorem 3.2). Assume that $Y_{1}$ has property (o) and $X$ satisfies the following condition: $\rho_{X}(x, y)=\rho_{Y}(x, y)$ for any two points $x$ and $y$ on the boundary $\partial Y$ of every $n$-dimensional compact connected $C^{0}$-submanifold $Y \subset X$ with $\partial Y \neq \varnothing$ satisfying (i), (ii), and (o). Suppose also that $\partial Y_{1}$ and $\partial Y_{2}$ are isometric in the metrics $\rho_{Y_{j}}$, where $j=1,2$. Then $\partial Y_{1}$ and $\partial Y_{2}$ are isometric in $\rho_{X}$.

Theorem 3.7. Let $n \geqslant 3$. Suppose that $(X, g)$ is an $n$-dimensional smooth complete connected Riemannian manifold without boundary and $Y_{1}$ and $Y_{2}$ are $n$-dimensional closed connected $C^{0}$ submanifolds with nonempty boundaries $\partial Y_{1}$ and $\partial Y_{2}$ in $X$ satisfying (i) and (ii). Assume that $\partial Y_{1}$ has property (o) and $X$ satisfies the following condition: $\rho_{X}(x, y)=\rho_{Y}(x, y)$ for any two points $x$ and $y$ on the boundary $\partial Y$ of every $n$-dimensional closed connected $C^{0}$-submanifold $Y$ with $\partial Y \neq \varnothing$ in $X$ satisfying (i), (ii), and (o). Suppose also that $\partial Y_{1}$ and $\partial Y_{2}$ are isometric in the metrics $\rho_{Y_{j}}(j=1,2)$. Then $\partial Y_{1}$ and $\partial Y_{2}$ are isometric in $\rho_{X}$.

Proofs of Theorems 3.2 and 3.4-3.7 are similar to the proof of Theorem 3.1 (Theorems 3.2 and 3.4-3.7 can be proved using the corresponding analogs of Theorems 2.1 and 2.3).

In conclusion, note that main results of our article were earlier announced in [1] and [2].

The authors were partially supported by the RFBR for, grants 14-01-00768-a and 15-0108275- $a$. 


\title{
References
}

[1] A.P.Kopylov, A rigidity condition for the boundary of a submanifold in a Riemannian manifold, Doklady Mathematics, 77(2008), no. 3, 340-341.

[2] A.P.Kopylov, Unique determination of domains, Differential Geometry and its Applications, Hackensack, NJ, World Sci. Publ., 2008, 157-169.

[3] A.V.Pogorelov, Extrinsic Geometry of Convex Surfaces, AMS, Providence, 1973.

[4] E.P.Sen'kin, Non-flexibility of convex surfaces, Ukr. Geom. Sb., 12 (1972), 131-152.

[5] A.P.Kopylov, Boundary values of mappings close to isometric mappings, Siberian Math. J., 25(1985), no. 3, 438-447.

[6] V.A.Aleksandrov, Isometry of domains in $\mathbb{R}^{n}$ and relative isometry of their boundaries, Siberian Math. J., 25(1985), no. 3, 339-347.

[7] V.A.Aleksandrov, Isometry of domains in $\mathbb{R}^{n}$ and relative isometry of their boundaries. II, Siberian Math. J., 26(1986), no. 6, 783-787.

[8] M.V.Korobkov, Necessary and sufficient conditions for unique determination of plane domains, Siberian Math. J., 49(2008), no. 3, 436-451.

[9] M.V.Korobkov, Some rigidity theorems in Analysis and Geometry, Dis. Dokt. Fiz.-Mat. Nauk, Novosibirsk, 2008 (in Russian).

[10] M.V.Korobkov, A criterion for the unique determination of domains in Euclidean spaces by the metrics of their boundaries induced by the intrinsic metrics of the domains, Siberian Advances in Mathematics, 20(2010), no. 4, 256-284.

[11] A.D.Aleksandrov, Intrinsic Geometry of Convex Surfaces, [English translation], Chapman \& Hall/CRC Taylor \& Francis Group, Boca Raton, 2006.

\section{Об условия жесткости границ подмногообразий риманового многообразия}

\author{
Анатолий П. Копылов \\ Михаил В. Коробков \\ Институт математики им. С. Л. Соболева СО РАН \\ пр. ак. Коптюга, 4, Новосибирск, 630090 \\ Пирогова, 2, Новосибирск, 630090 \\ Россия
}

\begin{abstract}
В прочессе развития идей академика А. Д. Александрова первым автором был предложен следующий подход к изучению проблем жесткости для краёв $C^{0}$-подмногообразий в некотором гладком римановом многообразии. Пусть $Y_{1}$ представляет собой двумерное компактное связное $C^{0}$ подмногообразие с непустым краем в некотором гладком двумерном римановом многообразии $(X, g)$ без края. Рассмотрим внутреннюю метрику (инфимум длин путей, соединяющих данную пару точек) внутренности Int $Y_{1}$ многообразия $Y_{1}$ и продолжим ее по непрерывности (операцией $\underline{\mathrm{lim}}$ ) на краевые точки $\partial Y_{1}$. В настоящей статье рассматривается вопрос о жесткости, т.е. когда указанная метрика определяет $\partial Y_{1}$ с точностъю до изометрии в обгемлющем пространстве $(X, g)$. Рассматривается также случай $\operatorname{dim} Y_{j}=\operatorname{dim} X=n, n>2$.
\end{abstract}

Ключевые слова: риманово многообразие, внутренняя метрика, индуцированная метрика на крае, строгая выпуклость многообразия, геодезические, условия жесткости. 